\title{
3. Augmented Intelligence - Wie Menschen mit KI zusammen arbeiten
}

\begin{abstract}
Moritz Kirste
KI-Technologien gelten in vielen Bereichen als bahnbrechend, da sie die kognitiven Leistungen des Menschen reproduzieren oder sogar übertreffen können. Menschliche Leistungen ließen sich somit durch KI ersetzen. Die Forschung im Bereich der Augmented Intelligence als Ergänzung zur KI geht jedoch davon aus, das sich menschliche und computergesteuerte kognitive Technologien im Idealfall positiv ergänzen. In diesem Beitrag wird die Motivation hinter der Forschung zur Augmented Intelligence erläutert und einige vielversprechende Ansätze ausgeführt.
\end{abstract}

Wofür haben Menschen Computer und Geräte wie Smartphones oder Tablets gebaut? Eine Antwort auf diese Frage lässt sich bei einem der ersten Computerpioniere, Konrad Zuse, in seinem autobiografischen Werk "Der Computer - Mein Lebenswerk" nachlesen: „Eine ausgesprochene Abneigung hatte ich gegen die statischen Rechnungen, mit denen man uns Bauingenieurstudenten quälte. Die Professoren, die diese Rechnerei beherrschten, bewunderte ich wie Halbgötter aus einer anderen Welt. Würde ich das jemals begreifen? Später sollte ich über das Problem des statischen Rechnens auf die Idee der programmgesteuerten Rechenmaschine kommen." (vgl. Zuse 1999, Springer 100 Jahre Zuse). Demnach wurde Zuse beim Bau seiner ersten Rechenmaschine davon motiviert, dass diese Maschine ihm intellektuell durchaus anspruchsvolle, aber lästige Denkarbeiten abnehmen sollte.

Es ist davon auszugehen, dass Zuse die so gewonnene Freiheit dafür nutzen wollte, Überlegungen nachzugehen, die ihm interessanter erschienen, während die Maschine die niederen Rechenaufgaben erledigte. Er dachte dabei auch schon an weiterführende Anwendungen, die über ein solches bloßes Berechnen, das im Grunde vergleichbar wäre mit einem besonders effektiven Abakus, hinausgingen: „Aber Zuse machte uns klar, daß Rechnen nur ein Spezialfall logischer Operationen ist und daß sein Apparat auch Schach spielen können müsse. Auch andere Anwendungsmöglichkeiten, wie Wettervorhersage,..." (vgl. Zuse 1999, Springer 100 Jahre Zuse). Dennoch wurden Computer lange Zeit hauptsächlich auf diese Weise eingesetzt, wie es die englische Bezeichnung „number cruncher" (Zahlenschieber) nahelegt. Beispielsweise wurde ENIAC (Electronic Numerical Integrator and Computer), der erste rein elektronische Universalrechner, für die Berechnung von ballistischen Tabellen für die US-Armee entwickelt. Aber auch die anderen ersten Rechenautomaten, sogenannte 
Berechner - das Wort Computer stammt vom lateinischen Wort für berechnen (computare) - wurden hauptsächlich in Bereichen eingesetzt, in denen die menschlichen Berechnungen zu aufwendig, schwierig oder langwierig waren wie Kryptografie, Wettervorhersagen, Simulationen von Atombombenexplosionen oder die Flugbahn von Raketen. Für die Programmierung und Steuerung dieser riesigen Rechenmaschinen wurden Lochkarten, Drehschalter und allererste Tastaturen genutzt. Was diese ersten Rechenautomaten jedoch nicht hatten, war etwas, das in unserer heutigen Nutzung aller elektronischen Geräte zentral ist: eine direktes Interface, also eine Steuerung mit einer visuellen oder sogar haptischen Bedienoberfläche.

Ohne ein Interface kann ein Computer nur in einem aufeinanderfolgenden stetigen Ablauf von Eingabe und Ausgabe genutzt werden. Heute werden Computer natürlich grundsätzlich anders genutzt. Es gibt beispielsweise eine Desktopoberfläche, die mit Maus und Tastatur gesteuert wird, beim Smartphone oder Tablet geschieht dies über Berührung, während die Auswirkungen direkt am Bildschirm sichtbar werden. Aber in vielerlei Hinsicht hat sich an der Nutzung ein Grundprinzip kaum verändert: Menschen benutzen die heutigen Geräte des Informationszeitalters als eine Form des kognitiven Outsourcings (Nielsen 2016), indem sie ein Problem oder eine Frage an das Gerät formulieren, das diese Problemstellung verarbeitet und eine Lösung präsentiert. Das geschieht in der Regel zwar über modernste Interfaces, die an das jeweilige Endgerät angepasst sind, der kognitiver Beitrag bleibt dabei jedoch häufig begrenzt und wird in einigen Fällen sogar aktiv beschränkt, um möglichst gleiche Ergebnisse zu erhalten. Ein Beispiel dafür ist die Wegfindung mit Hilfe eines Kartendienstes wie Google Maps oder Apple Maps. Der menschliche Nutzer fragt das Computerprogramm lediglich nach dem schnellsten Weg, ohne dass eine Rücksprache zur genaueren Intention stattfindet. In der Intention für den Weg könnten aber wichtige Informationen verborgen liegen, welche den idealen Weg beeinflussen. Es wäre beispielsweise denkbar, dass der Nutzer den Weg gerne mit einem ausgedehnten Spaziergang verbinden würde und insofern nicht am schnellsten, sondern angenehmsten Ergebnis interessiert ist.

Die aktuellen Debatten und Entwicklungen im Bereich der KI gehen häufig sogar noch einen Schritt weiter, denn vielfach wird davon ausgegangen, dass sich die kognitiven Leistungen des Menschen reproduzieren oder sogar ersetzen lassen. In diesem Sinne lesen sich die Meilensteine, an denen KI menschliche Leistungen übertroffen hat, als eine Geschichte des Sieges der KI über den Menschen und nicht als ein Versuch, die menschlichen Leistungen durch Computer zu verbessern: Schach 1997 (Campbell et al. 2002), Jeopardy 2011 (IBM 2010), Atari Computerspiele 2013 (Mnih et al.), Bilderkennung 2015 (He et al.), Spracherkennung 2015 (Amodei et al.) und Go 2016 (Silver et al. 2017). Ohne in die Debatte über die ethischen und gesellschaftlichen Konsequenzen dieser Entwicklung einzusteigen (siehe Teil C, Beitrag 12), 
drängt sich jedoch die zu Beginn formulierte Frage erneut auf: Wozu wollen Menschen Computer wirklich nutzen?

\section{Intelligence Augmentation}

Die Frage führt zurück auf einen Artikel von Douglas Engelbart (1962), „Augmenting Human Intellect: A Conceptual Framework". In seiner Vorstellung sollten Computer genutzt werden, die menschlichen Fähigkeiten zur Problemlösung zu verbessern. Engelbart war nicht nur der Erfinder der Computermaus, sondern seine Ideen sind bis heute sehr einflussreich im Bereich der Informatik, der Mensch-Maschine-Interaktion und der KI. Er prägte den Begriff der "Intelligence Augmentation" - augmentation lässt sich in diesem Falle mit Vergrößerung, Steigerung oder Erweiterung übersetzen - im Gegensatz zur "Artificial Intelligence”, also Kl. Die Idee geht davon aus, dass neue Konzepte der gemeinsamen Lösungsfindung von Mensch und Maschine gefunden werden müssen, die als Augmented Intelligence (Augmentierte Intelligenz), Intelligence Augmentation oder Artificial Intelligence Augmentation bezeichnet werden (Carter und Nielsen 2017). Die Intelligenz von Mensch und Maschine wird dabei synergetisch zur effizienteren Lösungsfindung eingesetzt. Der Vorteil dieser Synergie besteht darin, dass KI den Menschen nicht ersetzt, sondern ergänzt (IBM 2018). Gleichzeitig sind solche Systeme nur dann erfolgreich, wenn Mensch und KI über eine gemeinsame Sprache oder ein gemeinsames Dialogsystem verfügen, welches durch ein für alle Nutzer geeignetes Interface realisiert wird. Ein Nutzer muss in der Lage sein, Entscheidungen der KI vor ihrer Ausführung zu prüfen, um ggf. an neuralgischen Stellen des Prozesses eingreifen zu können. Dafür ist die Entwicklung neuer Schnittstellen notwendig, die es erlauben, die kritischen Stellen zu identifizieren. Die Nutzer müssen in den Ablauf des KI-Systems involviert sein, ohne dass ihre kognitive Belastung größer wird. Unter den Begriff Augmentation fallen auch physische Systeme wie beispielsweise Brain-Computer-Interfaces, Gehirnprothesen oder technische Werkzeuge zur Verbesserung der menschlichen Sinne. Im Beitrag werden ausschließlich die softwareseitigen Ansätze betrachtet, die an der Schnittstelle zwischen Augmented Intelligence und KI liegen.

Wie lässt sich Augmentierte Intelligenz vorstellen und wie lässt sie sich umsetzen? Ein einfaches Beispiel, von Carter und Nielsen (2017) übernommen, dient hier als Illustration: Das geometrische Gebilde eines Kreises lässt sich sowohl durch die Formel als auch durch die Zeichnung eines Kreises darstellen. Welche Darstellung ist besser? Welche enthält die wichtigen Informationen? Mit welcher kognitiven Transformation gelingt es uns besser, den Wesensgehalt eines Kreises zu erarbeiten, zu begreifen und für andere begreifbar zu machen (Nielsen 2016)? Augmentierte Intelligenz setzt genau an dieser Stelle an, indem sie uns dabei unterstützt, die jeweils passende kognitive Transformation und Repräsentation zu finden. Sie unterstützt auf diese Weise, 
den Inhalt unserer Gedanken selbst auf eine geeignete Metaebene zu transformieren und somit wiederum neue Erkenntnisse zu erlangen.

\section{Interaktives maschinelles Lernen}

Ein Ansatz für Augmented Intelligence ist das interaktive maschinelle Lernen (ML). Das Ziel dieses Verfahrens ist es, Menschen stärker in das jeweilige Lernverfahren des überwachten, unüberwachten und verstärkten Lernens einzubinden (siehe Einleitungskapitel „Entwicklungswege zur KI“). Für diese Idee eines "human-in-the-loop” gibt es eine Reihe von Beispielen (Amershi et al. 2014). Nutzer können dabei entweder vor oder nach einer Phase der Modellbildung durch den Lernalgorithmus einen gewissen Grad der Kontrolle und des Feedbacks übernehmen. Trainingsdaten können modifiziert werden, indem die Wichtigkeit bestimmter Merkmale hervorgehoben wird, Fehlertoleranzen können angepasst werden oder die durch den Algorithmus erkannten Muster und Strukturen können als passend oder unpassend bewertet werden. Solche Verfahren eignen sich insbesondere für Anwendungsfälle, bei denen die gewünschten Ergebnisse und Ausgaben, die der Lernalgorithmus aus den Trainingsdaten ausgeben soll, im Vorhinein noch nicht bekannt sind. Die Effizienz des Trainingsprozesses kann durch interaktives maschinelles Lernen zwar gesteigert werden, die Nutzer empfinden den Prozess aber oft als ermüdend oder langweilig, da sie in erster Linie nur für eine Verbesserung des Trainings benutzt werden, dabei aber kaum eigene kognitive Leistungen erbringen müssen (Amershi et al. 2014). Als Folge dessen gewinnen sie kaum wirkliches Wissen und Erkenntnisse, über die sie nicht auch schon vor der Nutzung des Computersystems verfügten.

\section{Visual Analytics und maschinelles Lernen}

Ein weiterer und vielversprechender Ansatz für Augmented Intelligence, der einige der Ideen des interaktiven ML zwar beinhaltet, aber weit darüber hinaus geht, ist die Kombination von maschinellem Lernen mit sogenannten Visual Analytics (Endert et al. 2017). Dabei werden große Datenmengen durch Methoden der KI und insbesondere des ML auf visuelle Art und Weise so aufbereitet, dass Menschen aus diesen Daten Erkenntnisse gewinnen können. Ein bekanntes Modell für diese Erkenntnisgewinnung stammt von Pirolli und Card (Pirolli und Card 2005). Es beschreibt den Prozess, wie aus Datenquellen Erkenntnisse erzeugt werden können. Das Modell unterteilt den Prozess in zwei Phasen: die erste sogenannte Hamsterphase, in der die ursprünglichen Informationen wiederholt auf Zusammenhänge durchsucht werden, und die zweite sogenannte Sinnstiftungsphase, in der die Informationen in ein Schema, überprüfbare Hypothesen und schlussendlich in eine präsentierbare Erkenntnis fließen. Systeme der Augmented Intelligence können Nutzer jetzt insbesondere in der Hamsterphase beim Durchforsten der Daten unterstützen. Der Nachteil an die- 
sem Modell ist der stark lineare Charakter, der von zunächst unstrukturierten Daten und Wissen hin zu einer Erkenntnis führt. Zudem wird kaum erklärt, wie ein Computer bei dieser Form der Wissensgenerierung unterstützen könnte.

Diese Nachteile versucht das Model von Sacha et al. (Sacha et al. 2014) auszugleichen (siehe Abbildung 3.1), indem es die Benutzung eines Computersystems in den menschlichen Erkenntnisprozess integriert. Es dient als Basis für einen iterativen Weg, der auch Vorkenntnisse berücksichtigt und erkenntnistheoretische Schlussverfahren wie Induktion, Deduktion und Abduktion einschließt (Ribarsky und Fisher 2016).

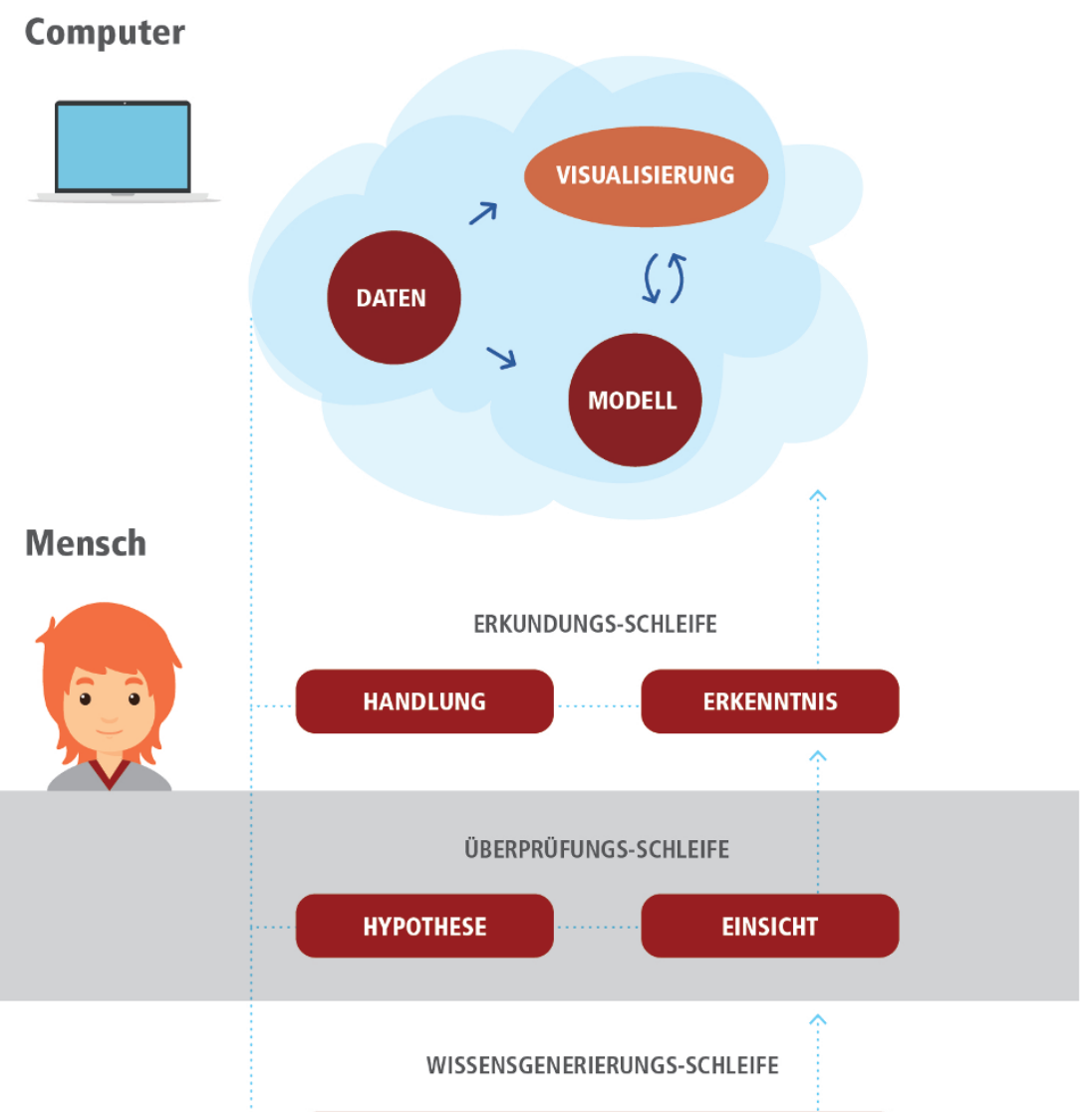

WISSEN

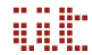

Abbildung 3.1: Das Modell von Sacha et al. zeigt die Integration eines Computersystems in den menschlichen Erkenntnisprozess. Wissen entsteht dabei durch wiederholte Schleifen innerhalb des Prozesses (eigene Darstellung adaptiert von Sacha et al. 2014). 
Unabhängig von der Modellierung des Erkenntnisprozesses erfolgt die Umsetzung der Kombination von Visual Analytics und maschinellem Lernen. Hierfür orientieren sich die Methoden an den vier Hauptverfahren Regression, Klassifikation, Clustering und Dimensionsreduktion (Einleitung Teil A). Interaktive Systeme erlauben die Modifizierung der verwendeten Algorithmen durch eine Anpassung der Parameter, der Vergleichswerte oder der zugrundeliegenden Algorithmen selbst. Dabei können die Nutzerinnen und Nutzer auf einer graphischen Benutzeroberfläche Datenpunkte, Variablen und Parameter sehen, Datenbereiche auswählen, Algorithmen nur auf diese Teilbereiche anwenden und sich im Anschluss die Auswirkungen auf die analysierten Daten anschauen. Ziel ist es, das Expertenwissen und die Algorithmen in Einklang zu bringen und im Falle einer Abweichung den Algorithmus möglichst effektiv anzupassen. Ein anschauliches Beispiel für diese Verfahren stammt aus einem Video der Firma Enlitic (Howard 2014). Hier werden Bilder anhand eines interaktiven Clusterverfahrens in wenigen Minuten sortiert, indem das Computersystem dem Nutzer immer wieder die Ergebnisse vorführt und dieser die Wichtigkeit und Unwichtigkeit bestimmter Merkmale hervorhebt. Die Zusammenarbeit erinnert an die Arbeit eines Forensikers, dem stets nur die relevanten Ergebnisse präsentiert werden und der dann darin Zusammenhänge erkennt.

\section{Dimensionsreduktion und GAN}

Ein Verfahren, das sich insbesondere um das Verständnis von Zusammenhängen bemüht, ist das der Dimensionsreduktion. Damit ist gemeint, dass ein hochdimensionaler Parameterraum durch einen kleineren Parameterraum möglichst so abgebildet wird, dass dabei keine Informationen verloren gehen. Dabei sind die Variablen des kleineren Parameterraums lineare Kombinationen oder nicht lineare Kombinationen der ursprünglichen Variablen. Die Verwendung lässt sich am Beispiel Bilderkennung anschaulich erklären. Für diese Anwendung werden Algorithmen des maschinellen Lernens, insbesondere Neuronale Netze, auf die Erkennung von Bildern trainiert. So können diese Netze aus vielen Bildern sehr treffsicher beispielsweise Hunde oder Gesichter erkennen (Einleitung Teil A). Im Prozess wird ein Bild und somit ein hochdimensionaler Raum, der aus Informationen über Position und Farbwert eines jeden Bildpunktes besteht, durch einen kleineren Raum abgebildet, der nur die Information enthält, was auf dem Bild zu sehen ist. Das komplexe hochdimensionale Bild wird auf eine einfachere Information reduziert, die beispielsweise nur abbildet, ob es sich um einen Hund oder Elefanten handelt (siehe Abbildung 3.2).

Man kann diesen Prozess auch umkehren und herausfinden, welche Merkmale eines Bildes denn einen Hund ausmachen oder charakterisieren, beziehungsweise aus welchen Parametern welches Hundebild entsteht. Hierfür sind insbesondere die GAN sehr hilfreich, da sie helfen, diejenigen Eingabeparameter zu entdecken, die ein 


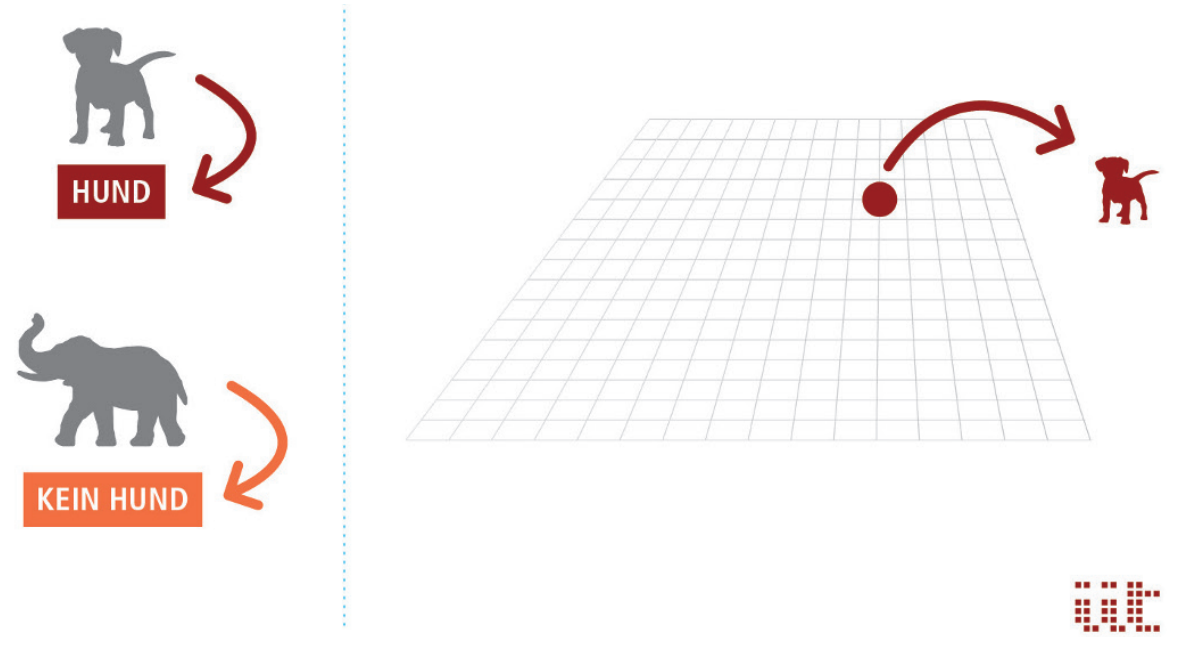

Abbildung 3.2: Dimensionsreduktion: Durch ein Neuronales Netz wird ein hochdimensionaler Raum, hier der Inhalt eines Bildes, auf einen kleineren Raum abgebildet (links). Bei einem GAN kann ein niederdimensionaler Raum der Hundemerkmale dargestellt werden, in welchem jeder Punkt einer bestimmten Ausprägung eines Hundes entspricht (rechts) (eigene Darstellung in Anlehnung an Carter und Nielsen 2017).

bestimmtes Bild charakterisieren. Das ist anders als ein klassisches neuronales Netz, das nach einer Trainingsphase an bekannten Bildern ein neu zu bestimmendes Bild erkennen kann. Durch diese Umkehrung entsteht ein niederdimensionaler Raum der Hundemerkmale, in welchem jeder Punkt einer bestimmten Ausprägung eines Hundes entspricht (siehe Abbildung 3.2). Welche Vorteile sich daraus für das Verständnis von Zusammenhängen ergeben, wird deutlich, da der weniger dimensionale Raum mit menschlichen Begriffen beschreibbar und für Menschen nachvollziehbar gestaltet werden kann - Beinanzahl, Schwanzlänge, Haarfarbe, Maulgröße usw. - und eine Veränderung der Parameter in diesem Raum hat die unmittelbare Änderung des entsprechenden Hundebildes zur Folge.

Wie könnte ein Interface für ein solches System aussehen? Für das Beispiel des Hundebildes hieße das, eine Vielzahl von Bildern verschiedener Hunderassen zu charakterisieren. Im niederdimensionalen Raum der Hundemerkmale sollten Hunde der gleichen Rasse jeweils in einem ähnlichen Bereich landen, während Hunde anderer Rassen räumlich getrennt wären (siehe Abbildung 3.3). Durch eine Verbindung mathematisch wäre dies ein Vektor - zwischen dem Zentrum des einen Bereichs und dem Zentrum des anderen Bereiches, ergibt sich eine gute Möglichkeit, Hundemischlinge zwischen diesen beiden Rassen zu charakterisieren, deren Merkmale sich ent- 
lang dieses sogenannten Attributevektors bewegen. Ein Interface für Augmented Intelligence könnte nun einerseits die Dimensionsreduktion ausführen, die Bereiche der Gemeinsamkeiten anzeigen, Verbindungen ziehen und die Manipulation der Daten entlang dieser Verbindungen erlauben. Diese Idee eines Interface, welches ein tieferes Verständnis ermöglicht, lässt sich auch auf andere Zusammenhänge übertragen, in denen ein hochdimensionaler Datenraum, der sich von Menschen nur schwer durchsuchen lässt, auf einen deutlich niedrigeren Dimensionsraum abgebildet werden kann.

Die Idee der Verbindungsvektoren ist nur eine und im Grunde auch sehr simple Methode, die dabei helfen kann, Zusammenhänge in den Daten zu erkennen und für weitere Erkenntnisse zu nutzen. Eine weitere Möglichkeit lässt sich ebenfalls anhand eines Beispiels illustrieren: Dabei wird eine riesige Anzahl von Kochrezepten mit einem GAN analysiert und auf einen niederdimensionalen Raum abgebildet. In diesem Raum könnte der Schärfegrad der Gerichte ein wichtiger Parameter sein. Ein Nutzer kann ein bestimmtes Gericht im Kopf haben, das durch einen Punkt in diesem Parameterraum dargestellt wird, und einen bestimmten Schärfegrad anstreben, der durch eine Linie im Parameterraum wiedergegeben wird (siehe Abbildung 3.4). Ein Interface für Augmented Intelligence erlaubt es dem Nutzer nun, diese Linie und den Punkt zu bestimmen. Das System zeigt dann die kürzeste Verbindung zwischen Linie

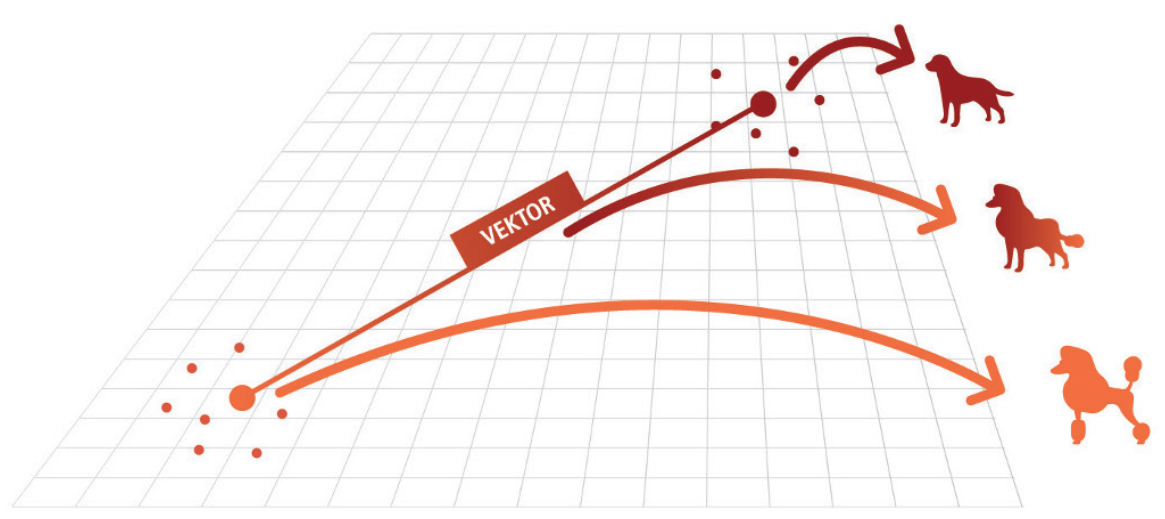

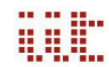

Abbildung 3.3: Die Abbildung veranschaulicht die Funktion eines Attributevektors, der zwischen dem Schwerpunkt einer Hunderasse (Labradore) in den Schwerpunkt einer anderen Hunderasse (Poodle) zeigt. Auf dem Vektor läge dann ein Mischling zwischen den beiden Rassen (Labradoodle) (eigene Darstellung in Anlehnung an Carter und Nielsen 2017). 


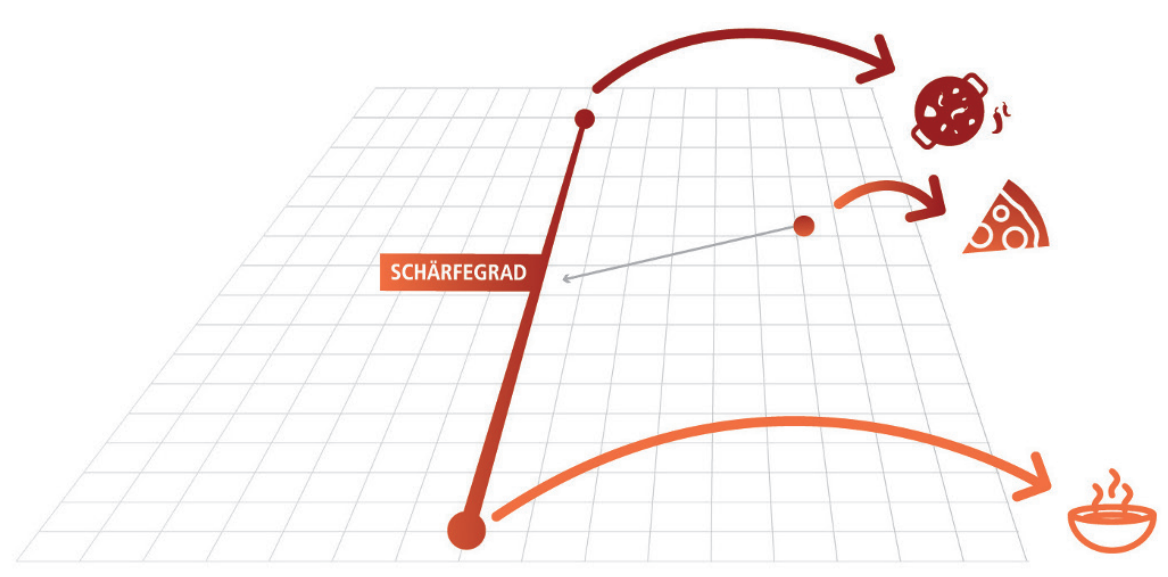

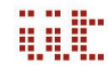

Abbildung 3.4: Datenreduktion: Jeder Punkt im Raum entspricht einem Gericht. Der Schärfegrad lässt sich als Linie zwischen einem sehr scharfen (Chilli) und einem nicht scharfen Gericht (Gemüsesuppe) darstellen. Die Parameter des gewünschten Gerichtes (Pizza) können jetzt so variiert werden, dass sie einem bestimmten Schärfegrad entsprechen (eigene Darstellung in Anlehnung an Carter und Nielsen 2017).

und Punkt an und welche Parameter (z. B. Zutaten und Mengen) des geplanten Gerichtes modifiziert werden müssen, damit sie dem gewünschten Schärfegrad entsprechen. Dieses Bewegen eines Punktes innerhalb gewisser Grenzen des niederdimensionalen Parameterraums kann ebenso wie die zuvor beschriebene Methode zu neuen Erkenntnissen führen, da sich die Auswirkungen im hochdimensionalen Raum - in diesem Fall also das modifizierte Gericht - direkt beobachten ließen.

Sowohl die Hundebilder als auch die Kochrezepte sind natürlich sehr einfache Beispiele, von denen sich schwerlich behaupten ließe, dass ein Interface für Augmented Intelligence hier wirklich neue Einblicke und Erkenntnisse liefern kann. Ganz anders stellt sich dies in einem weiteren Szenario dar, in dem deutlich wird, welche Vorteile ein funktionierendes Interface, das Visual Analytics und ML kombiniert, bieten könnte: In einem Chemielabor arbeitet eine Chemikerin an der Untersuchung der möglicherweise umweltschädlichen Auswirkungen von Inhaltsstoffen in Unkrautvernichtungsmitteln, die in der Landwirtschaft eingesetzt werden sollen. Für eine Entscheidungsfindung hat sie eine Vielzahl an Möglichkeiten, sie verfügt aber nur über begrenzte finanzielle Mittel. Außerdem muss sie die Entscheidung schnell treffen, da ansonsten die Zulassung des Mittels auf dem Spiel steht. Die Chemikerin kann auf 
große Datenbanken, die Wissen über chemische Stoffe enthalten, zurückgreifen, sie könnte eigene Feld- und Labortest durchführen, um die Auswirkungen zu analysieren, oder sie könnte die exakte chemische Zusammensetzung des Mittels herausfinden. Ein Augmented Intelligence-System soll ihr bei der Entscheidung helfen. Zunächst stellt das System alle bisher vorhanden Informationen visuell dar. Die Chemikerin beginnt diese Informationen zu ordnen, indem sie bestimmten Inhalten gröBere Wichtigkeit zuweist als anderen (Visual Analytics). Das System passt seine Ordnung mittels ML direkt an und stellt die Inhalte neu dar. Auf diese Weise erkennt die Chemikerin, welche Tests einen großen Informationsgewinn ermöglichen und welche Tests nur bereits bekanntes Wissen reproduzieren - hier könnte die Dimensionsreduktion durch ein GAN helfen. Das System macht eigene Vorschläge für sinnvolle Tests, indem es den zu erwartenden Erkenntnisgewinn zeigt, und adaptiert die dargestellten Inhalte und Ordnungsstruktur direkt an die neuen Ergebnisse. Ziel ist, den Raum aller Informationen und Kombinationsmöglichkeiten nicht komplett zu durchdringen, sondern nur einen sinnvollen Pfad zu einem Ergebnis zu beschreiten. Am Ende wird die komplexe Fragestellung effizient und von Menschen nachvollziehbar beantwortet.

Bis ein solches Szenario realisierbar ist, müssen die bisherigen Ansätze weiter erforscht und entwickelt werden. Ein Aspekt ist hervorzuheben - das Verständnis natürlicher Sprache durch Computersysteme. Dies ist deshalb so wichtig, weil Menschen ständig erklären, zuhören und generell kommunizieren und auf diese Weise nicht nur Erkenntnisse gewinnen, sondern auch (mit-)teilen. Für eine Augmented Intelligence ist es entscheidend, diesen Informationskanal zu verstehen und nutzen zu können. Wie groß die Bedeutung ist, sieht man an den aktuellen Erfolgen mit smarten Lautsprechern im Consumerbereich (Statista und Brandt Mathias). Erst als sich die Kommunikation mit der $\mathrm{KI}$ in diesen Systemen für Menschen natürlich anfühlte, konnten die Systeme erfolgreich werden. Neue Entwicklungen auf diesem Gebiet deuten darauf hin, dass sich diese Systeme in den kommenden Jahren deutlich verbessern werden (Hirschberg und Manning 2015).

\section{KI-Akzeptanz}

Weitere Aspekte, die den Umgang mit Augmented Intelligence und KI im Allgemeinen maßgeblich beeinflussen, sind Vertrauen, Verständnis und Erklärbarkeit von Entscheidungen. Es ist anzunehmen, dass die Akzeptanz von KI verbessert werden kann, wenn deren Analysen und Vorschläge für Menschen erklärbar und damit auch nachvollziehbar sind und auf diese Weise Vertrauen schaffen. Eine allgemein verständliche und nutzbare Schnittstelle für Augmented Intelligence kann dazu dienen, die Nachvollziehbarkeit und das Vertrauen in KI zu stärken. Die Wichtigkeit dieser Aspekte zeigen sich in der aktuellen DARPA Challenge (DARPA), bei der Computer- 
systeme entwickelt werden, die solche potenziellen Fragen eines Nutzers beantworten sollen, um zu einem besseren Verständnis zu gelangen (siehe Abbildung 3.5).

Es stellt sich bei der Entwicklung der beschriebenen Systeme für Augmented Intelligence natürlich die Frage, ob eine Verbesserung der kognitiven Leistungen über-

\section{KI HEUTE}

\section{VERSTÄNDLICHE KI}

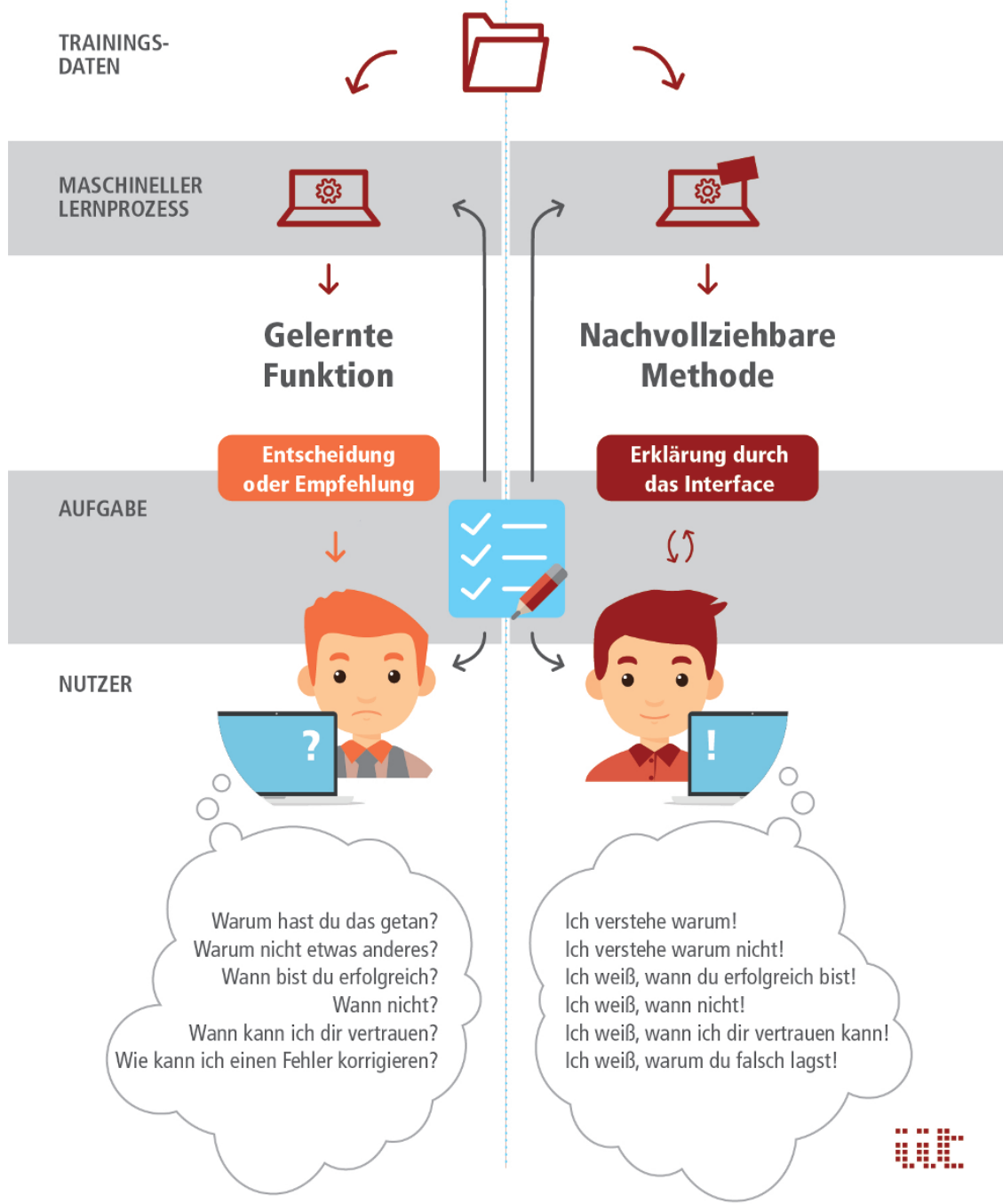

Abbildung 3.5: Darstellung des Modells eines für Nutzende nachvollziehbaren Systems der KI (eigene Darstellung in Anlehnung an DARPA 2018). 
haupt messbar ist. Und wenn ja, wie? Lässt sich eventuell eine Art Intelligenztest überlegen, der die Zusammenarbeit von Mensch und KI bewertet und insbesondere nachweist, dass diese Zusammenarbeit ungleich fruchtbarer ist, als wenn wir menschliche durch KI ersetzten? Von der Antwort auf die Frage hängt es ab, welche Computersysteme in den kommenden Jahren entwickelt werden und ob diese, um auf das anfängliche Zitat von Konrad Zuse zurückzukommen, uns Freiheit schaffen oder im schlimmsten Falle diese sogar einschränken. 


\section{Literatur}

Amershi, Saleema; Cakmak, Maya; Knox, W. Bradley; Kulesza, Todd (2014): Power to the People. The Role of Humans in Interactive Machine Learning. In: Al Magazine. Online verfügbar unter https://www.microsoft.com/en-us/research/publication/power-to-thepeople-the-role-of-humans-in-interactive-machine-learning/. Zuletzt geprüft am 23.02.2018.

Amodei, Dario; Anubhai, Rishita; Battenberg, Eric; Case, Carl; Casper, Jared; Catanzaro, Bryan et al.: Deep Speech 2. End-to-End Speech Recognition in English and Mandarin. Online verfügbar unter http://arxiv.org/pdf/1512.02595v1. Zuletzt geprüft am 23.02.2018.

Campbell, Murray; Hoane, A.Joseph; Hsu, Feng-hsiung (2002): Deep Blue. In: Artificial Intelligence 134 (1-2), S. 57-83. DOI: 10.1016/S0004-3702(01)00129-1.

Carter, Shan; Nielsen, Michael (2017): Using Artificial Intelligence to Augment Human Intelligence. In: Distill 2 (12). DOI: 10.23915/distill.00009.

DARPA: Explainable Artificial Intelligence (XAI). Online verfügbar unter https://www.darpa. mil/program/explainable-artificial-intelligence, zuletzt geprüft am 23.02.2018.

Endert, A.; Ribarsky, W.; Turkay, C.; Wong, B. WilliamL.; Nabney, I.; Blanco, I. Díaz; Rossi, F. (2017): The State of the Art in Integrating Machine Learning into Visual Analytics. In: Computer Graphics Forum 36 (8), S. 458-486. DOI: 10.1111/cgf.13092.

Engelbart, Douglas Carl (1962): Augmenting Human Intellect: A Conceptual Framework.

He, Kaiming; Zhang, Xiangyu; Ren, Shaoqing; Sun, Jian: Deep Residual Learning for Image Recognition. Online verfügbar unter http://arxiv.org/pdf/1512.03385v1. Zuletzt geprüft am 23.02.2018.

Hirschberg, Julia; Manning, Christopher D. (2015): Advances in natural language processing. In: Science (New York, N.Y.) 349 (6245), S. 261-266. DOI: 10.1126/science.aaa8685.

Howard, Jeremy (2014): The wonderful and terrifying implications of computers that can learn. Online verfügbar unter https://www.ted.com/talks/jeremy_howard_the_wonderful_ and_terrifying_implications_of_computers_that_can_learn?language=en, zuletzt geprüft am 23.02.2018.

IBM: Response to - Request for Information Preparing for the Future of Artificial Intelligence. IBM. Online verfügbar unter https://www.research.ibm.com/cognitive-computing/ostp/ rfi-response.shtml, zuletzt geprüft am 23.02.2018.

Mnih, Volodymyr; Badia, Adrià Puigdomènech; Mirza, Mehdi; Graves, Alex; Lillicrap, Timothy P.; Harley, Tim et al.: Asynchronous Methods for Deep Reinforcement Learning. Online verfügbar unter http://arxiv.org/pdf/1602.01783v2, zuletzt geprüft am 23.02.2018.

Nielsen, Michael (2016): Thought as a Technology. Online verfügbar unter http://cognitivemedium.com/tat/, zuletzt geprüft am 23.02.2018. 
Pirolli, Peter; Card, Stuart K. (2005): The sensemaking process and leverage points for analyst technology as identified through cognitive task analysis. Proceedings of International Conference on Intelligence Analysis.

IBM (2010): IBM's "Watson" Computing System to Challenge All Time Greatest Jeopardy! Champions. Online verfügbar unter https://www-03.ibm.com/press/us/en/pressrelease/33233.wss, zuletzt geprüft am 23.02.2018.

Ribarsky, William; Fisher, Brian (2016): The Human-Computer System. Towards an Operational Model for Problem Solving. In: 2016 49th Hawaii International Conference on System Sciences (HICSS). 2016 49th Hawaii International Conference on System Sciences (HICSS). Koloa, HI, USA, 05.01.2016 - 08.01.2016: IEEE, S. 1446-1455.

Sacha, Dominik; Stoffel, Andreas; Stoffel, Florian; Kwon, Bum Chul; Ellis, Geoffrey; Keim, Daniel A. (2014): Knowledge Generation Model for Visual Analytics. In: IEEE transactions on visualization and computer graphics 20 (12), S. 1604-1613. DOI: 10.1109/ TVCG.2014.2346481.

Satista; Brandt Mathias: Smart Speaker, Wenig Echo in Deutschland. Online verfügbar unter https://de.statista.com/infografik/12884/smart-speaker-besitz-in-deutschland-und-denusa/, zuletzt geprüft am 23.02.2018.

Silver, David; Schrittwieser, Julian; Simonyan, Karen; Antonoglou, loannis; Huang, Aja; Guez, Arthur et al. (2017): Mastering the game of Go without human knowledge. In: Nature 550 (7676), S. 354-359. DOI: 10.1038/nature24270.

\section{(c) (i)}

Dieses Kapitel wird unter der Creative Commons Namensnennung 4.0 International Lizenz http://creativecommons.org/licenses/by/4.0/deed.de) veröffentlicht, welche die Nutzung, Vervielfältigung, Bearbeitung, Verbreitung und Wiedergabe in jeglichem Medium und Format erlaubt, sofern Sie den/die ursprünglichen Autor(en) und die Quelle ordnungsgemäß nennen, einen Link zur Creative Commons Lizenz beifügen und angeben, ob Änderungen vorgenommen wurden.

Die in diesem Kapitel enthaltenen Bilder und sonstiges Drittmaterial unterliegen ebenfalls der genannten Creative Commons Lizenz, sofern sich aus der Abbildungslegende nichts anderes ergibt. Sofern das betreffende Material nicht unter der genannten Creative Commons Lizenz steht und die betreffende Handlung nicht nach gesetzlichen Vorschriften erlaubt ist, ist für die oben aufgeführten Weiterverwendungen des Materials die Einwilligung des jeweiligen Rechteinhabers einzuholen. 